\title{
1 Chondrocyte cell adhesion on chitosan supports using single- 2 cell Atomic Force Microscopy
}

3 Christian Enrique García García, ${ }^{* a, b}$ Claude Verdier, ${ }^{*}{ }^{c}$ Bernard Lardy, ${ }^{\mathrm{d}}$ Frédéric

4 Bossard, ${ }^{\mathrm{b}}$ Félix Armando Soltero Martínez ${ }^{\mathrm{a}}$ and Marguerite Rinaudo ${ }^{\mathrm{e}}$

5 a Universidad de Guadalajara, Departamento de Ingeniería Química. Blvd. M. García Barragán

$6 \# 1451$, C.P. 44430, Guadalajara, Jalisco, México; christian-enrique.garcia-garcia@univ-

7 grenoble-alpes.fr; jfasm@ @otmail.com

8 b Univ. Grenoble Alpes, CNRS, Grenoble INP**, LRP, 38000 Grenoble, France **Institute of

$9 \quad$ Engineering Univ. Grenoble Alpes; frederic.bossard@univ-grenoble-alpes.fr

$10{ }^{\mathrm{c}}$ Univ. Grenoble Alpes, CNRS, LIPHY, F-38000 Grenoble, France

11 d Pôle Biologie, DBTP, Biochimie des Enzymes et des Protéines, CHU-Grenoble, 38000

12 Grenoble, France ; blardy@ chu-grenoble.fr

e Biomaterials Applications, 6 Rue Lesdiguières, 38000 Grenoble, France. marguerite.rinaudo38@gmail.com

$\dagger$ Corresponding Author

* Claude Verdier, claude.verdier@univ-grenoble-alpes.fr,

Tel: +33476635980

\section{Abstract}

In this work, functional scaffolds based on electrospun chitosan nanofibers are studied in terms of their cell adhesion response. To prove cell compatibility of this biomaterial, chondrocyte interactions are investigated using atomic force microscopy in which a single cell is fixed to the cantilever and approached to the chitosan mat for a given contact time. Then, the cantilever is retracted and cell interactions are observed. Force jumps distribution for cell detachment is described and the adhesion energy is determined comparing nanofibers mats with homogeneous films and a BSA coated surface as control. Force adhesion on chitosan film equals $460 \mathrm{pN}$ slightly higher than porous fiber mat (410 $\mathrm{pN}$ ) indicating that more cell-substrate bonds could be formed on a flat contact surface. The adhesion on hydrophilic chitosan surface is much larger than on the control surface $(210 \mathrm{pN})$ due to its positive character and ability for H-bond stabilization.

Key words: AFM, cell adhesion, chondrocyte, electrospun chitosan substrate, detachment energy

\section{Introduction}

One of the most studied biological applications of electrospun materials consists in the replacement and regeneration of damaged human tissue. Cells are supposed to use the artificial mat as a scaffold to adhere, adapt and proliferate in order to repair the injured tissue (mainly connective tissue, articular cartilage, joints and bones) affected by common diseases such as arthritis, osteoarthritis, arthropathy, joint dysfunction or after a surgery (Changhsun et al., 2008; Yamane et al., 2005). In the case of articular cartilage disorders, chirurgical interventions remain the viable option since no long-term efficient 
pharmacological treatments are proposed yet (Huang, Hu, \& Athanasiou, 2016; Rai, Dilisio, Dietz, \& Agrawal, 2017). These invasive treatments include the extraction of the injured tissue followed by its artificial replacement in order to disrupt the progression of the illness (Changhsun et al., 2008; Nguyen \& Gu, 2016).

With the aim of a solution for this problematic, several approaches have been proposed in the literature concluding that research about biological properties must include the investigation of cell adhesion and compatibility with the new polymeric scaffold (Huang et al., 2016; Rai et al., 2017; Yamane et al., 2005).

In tissue engineering, all factors influencing the behavior of cells dealing with new tissue replacements are important. The study of cell-to-scaffold adhesion could lead to a better understanding of many biological processes, especially cell migration, differentiation and proliferation (Huang et al., 2016; Titushkin \& Cho, 2006). These processes are related to the cell response to their microenvironment which includes nutriment and growth factor concentrations as well as substrate morphology (chemo-mechanical properties) (Cohen, Klein, Geiger, \& Addadi, 2003; Nguyen \& Gu, 2016; Varady \& Grodzinsky, 2016). On this point, it has been shown that fiber mats, obtained by electrospinning, with low density and high porosity are well adapted for cell development (Soliman et al., 2011).

Cell adhesion is the ability of a cell to stick to a surface that could be another cell, the extracellular matrix (ECM) or different scaffolds (bulk material, gels) (Puech et al., 2005). Numerous strategies and methods have been proposed and performed to quantify this interaction between cells and their environment. Laser Optical Tweezers (LOT) and Atomic Force Microscopy (AFM) are important tools for the quantification and understanding of biological sample properties at the micro and nanoscale (Changhsun et al., 2008; Iscru, Anghelina, Agarwal, \& Agarwal, 2008; Nguyen \& Gu, 2016; Titushkin \& Cho, 2006; Ungai-Salánki et al., 2019; Whitehead, Rogers, Colligon, Wright, \& Verran, 2006).

The AFM technique allows to characterize either normal cell-substrate interactions, cellcell adhesion and lateral cell detachment (Nguyen \& Gu, 2016). Some of the studied mechanisms to measure cell adhesion strength, applying AFM, include the approach onto an adhered cell to measure adhesion force between the cell and a functionalized cantilever tip (Changhsun et al., 2008). Measurement of adhesion force between two cells has been also performed by using cells attached to the cantilever and brought into contact with another adherent cell. Finally, AFM cantilever has been used to apply a shear force on a cell until it is detached; tangential adhesion force between the cell and the substrate is then measured (Nguyen \& Gu, 2016).

In the particular case of chondrocytes, being related with cartilage tissue reconstruction (Changhsun et al., 2008; Nguyen \& Gu, 2016), AFM helped to measure the cell response to normal and lateral external forces. AFM has also facilitated the understanding of how a disease can affect the adhesion properties and stiffness of cells. It has been reported that chondrocytes can resist normal forces better than tangential shear in the early steps of the adhesion process when adhesion molecules establish early attachment to substrates. When cells spread, strong adhesion is generated leading to higher detachment tangential shear through the formation and action of stress fiber bundles (Nguyen \& Gu, 2016). 
The method known as single-cell force spectroscopy (SCFS) consists in the immobilization of a single living cell on an AFM cantilever and the measurement of the interaction forces between the cellular entity and a bio-interface, which can be a tissue, another cell or a surface (Puech et al., 2005; Ungai-Salánki et al., 2019). In SCFS, the cell attached to the cantilever is pushed until contact with the substrate or to the other cell, allowing direct measurement of cell-surface or cell-cell adhesion, respectively. Since both spatial resolution and force sensitivity are high, the AFM was the first method able to measure cell adhesion (Puech et al., 2005; Ungai-Salánki et al., 2019).

In many studies, experiments involving the lateral force detachment of chondrocytes using AFM were applied in order to study the adhesive force response between cells and the substrate. In these assays, high lateral force values were observed. For instance, in the case of single living chondrocytes seeded during 3,6 and 24 hours on a Petri dish, the adhesion force increases from $74.14 \pm 13.81 \mathrm{nN}$ to $171.02 \pm 34.24 \mathrm{nN}$ then to $185.48 \pm$ $39.50 \mathrm{nN}$, respectively (Nguyen $\& \mathrm{Gu}, 2016$ ). Other experiments on cervical carcinoma cells were performed to investigate the effect of various adsorbed proteins on polystyrene substrates usually used for cell culture. The determined adhesion forces varied from 20 to $200 \mathrm{nN}$, increasing with time and temperature; higher values were observed on hydrophilic substrates compared to hydrophobic supports, as a function of the adsorbed protein content (Sagvolden, Giaever, Pettersen, \& Feder, 1999). An original technique was used to measure the strong adhesion of Caulobacter crescentus to a solid substrate with a suction flexible glass micropipette adapted for measuring forces ranging from tens of $\mathrm{nN}$ to tens of $\mu \mathrm{N}$. Values around $0.59 \mu \mathrm{N}$ were found for the detachment force (Tsang, Li, Brun, Freund, \& Tang, 2006).

Chitosan-based materials, especially developed for biomedical applications, have attracted attention for a long time. In terms of practicality and efficiency, non-woven membranes of electrospun nanofibers are well known for their porous structures and relatively large surface area, which provide ideal materials to mimic the natural extracellular matrix (ECM) (Ribba, Parisi, D’Accorso, \& Goyanes, 2014). One advantage of chitosan, a pseudo-natural polymer, is that it becomes water soluble in acidic conditions due to $-\mathrm{NH}_{2}$ protonation as soon as its degree of acetylation is lower than 0.5 ; then, chitosan solution processing is relatively easy. Chitosan is an interesting biodegradable and biocompatible polymer with hemostatic properties, anti-inflammatory response, antibacterial and antifungal properties often described in the literature, and is well adapted for biological applications (Sapkota \& Chou, 2020; Younes \& Rinaudo, 2015). In addition, chitosan is stabilized by the H-bond network in the solid state, providing good mechanical properties under film or fibrous materials.

Chitosan scaffolds produced by electrospinning, as an approach of a developed advanced technology to improve tissue engineering, are proposed in this research work. Being a natural based polymer and considering its particular properties such as biodegradability and non-toxicity, its application is outstanding as compared to other polymers and biopolymers. Nevertheless, the degree of acetylation of chitosan samples applied for cell development must be preferentially lower than 0.13 (Amaral, Cordeiro, Sampaio, \& Barbosa, 2007). 
In order to show the effect of the electrospun chitosan mats topography on cell adhesion, force measurements at the nanoscale provided by the AFM technique have been performed using the SCFS method. Helping to demonstrate the advantage of using chitosan-based fibers to improve chondrocyte cell adhesion in tissue engineering applications, the results are compared with the adhesion response on chitosan films as well as a BSA-coated Petri dish surface.

Based on the hypothesis that chitosan substrates are able to supply an appropriate environment for chondrocyte adhesion, culture and phenotype maintaining, AFM measurements will help understand and validate the choice of biomaterials from this source. Single cells fixed on the cantilever interact directly with the support allowing to quantify the force and the energy needed to separate the cell from the substrate. At the same time, analogous results can be expected between the 2 studied substrate morphologies (film and fiber) even though, chitosan fiber mats are preferable biomaterials. Adhesion energy is favored by the $\mathrm{H}$-bond stabilization and possible charge interaction on chitosan supports.

\section{Experimental}

\subsection{Materials}

The chitosan (CS) sample was from Northern cold-water shrimp, Pandalus borealis, from Primex Ehf (Batch TM4778, code 42010, Siglufjordur, Iceland), with a molecular weight $\left(M_{\mathrm{W}}\right)$ around $160 \mathrm{~kg} / \mathrm{mol}$ and a degree of acetylation (DA) of 0.05 , determined using ${ }^{1} \mathrm{H}$ NMR. Poly (ethylene oxide) (PEO) with a molecular weight of $1 \times 10^{3} \mathrm{~kg} / \mathrm{mol}$ was used to prepare the fibrous mat. Acetic acid ( $\geq 99.7 \%)$ was used as solvent for both polymers, ethanol and $\mathrm{K}_{2} \mathrm{CO}_{3}$ were purchased from Sigma-Aldrich (France). Deionized water was used to prepare the solutions. All reagents and polymers were used as received without further purification.

For cell culture, the C-20/A4 human chondrocyte cell line (Goldring et al., 1994) was selected and the samples were seeded in Dulbecco's Modified Eagle Medium (DMEM) supplemented with $10 \% \mathrm{v} / \mathrm{v}$ of fetal bovine serum (FBS) and a $1 \% \mathrm{v} / \mathrm{v}$, in proportion with the total volume, penicillin/streptomycin/glutamine solution. Phosphate Buffered Saline (PBS) solution with a $\mathrm{pH}=7.4$, measured in the laboratory, DMEM serum-free and $0.05 \%$ Trypsin-EDTA solution were also utilized in cell experiments. All biological reagents were purchased from Gibco Life Technologies (Paisley, UK).

\subsection{Cell culture}

The initial sample of chondrocytes C-20/A4 was disposed in a culture flask, $20 \mathrm{~mL}$ of complete DMEM were added and the sample was preserved into a cell incubator (inCu safe, Panasonic) at $37{ }^{\circ} \mathrm{C}$ and $5 \% \mathrm{CO}_{2}$ constant inlet flow during few days until confluence.

Before AFM tests, trypsinization was used for cell detachment from culture flask; a final cell suspension was prepared in complete DMEM containing around $1 \times 10^{6}$ cell $/ \mathrm{mL}$.

\subsection{Engineering CS scaffolds by electrospinning}

Chitosan nanofibers were produced by electrospinning of the CS/PEO system at 70/30 $(\mathrm{w} / \mathrm{w})$ proportion related to the total polymer concentration $(5 \% \mathrm{w} / \mathrm{w})$. The blend was 
prepared in $0.5 \mathrm{M}$ acetic acid and the nanofibrous scaffolds were fabricated using a conventional vertical electrospinning arrangement, as described elsewhere (Garcia, Soltero Martínez, Bossard, \& Rinaudo, 2018).

Nanofibers were collected on an aluminum foil and the so-formed-mats were left at ambient conditions to evaporate the excess of acetic acid and water, prior to further analyses.

\subsection{Preparation of Chitosan films by evaporation casting}

A constant amount ( 1.0 gram) of a $5 \% \mathrm{w} / \mathrm{w}$ CS solution was placed in a Teflon mold of known volume to obtain a uniform polymer film with a thickness between $40-50 \mu \mathrm{m}$ (measured with a Mitutoyo Digimatic micrometer, with precision of $0.001 \mathrm{~mm}$ ). The probes were stored at room temperature for 3 days until complete evaporation of the solvent.

\subsection{Chitosan Neutralization}

Spun nanofiber mats and CS casted films were weighted and cut at the desired size before being immersed in alkaline ethanol/water 70/30 v/v mixture, prepared by dissolving $\mathrm{K}_{2} \mathrm{CO}_{3}$ until achieving a basic $\mathrm{pH}$ value around 12 . This step helps to neutralize the protonated chitosan amino groups when CS is dissolved in acidic conditions (Rinaudo, 2006). Further, CS films and nanofiber mats were washed for 3 days, four times a day, with deionized water until $\mathrm{pH}$ neutralization, to remove the salt formed from chitosan solutions (potassium acetate) and $\mathrm{K}_{2} \mathrm{CO}_{3}$ excess. Finally, the membranes were dried at room temperature before being used in AFM measurements.

\subsection{Substrate fixing}

Substrate samples, covering the majority of the circular surface $\left(9.2 \mathrm{~cm}^{2}\right)$ of the culture Petri dish (Techno-Plastic product AG, Switzerland), were selected.

UV curing NOA 68, Norland Optical Adhesive 68 (Lot 319, Norland Products, INC, Cranbury, NJ, USA), was used to stick the solid substrates to the bottom part of the culture dish. Different adhesion points were created by putting a small amount of the product between the substrate and the dish; NOA 68 was left acting during 15 minutes under UV radiation before AFM tests.

In order to have a reference surface for the adhesion response, a culture dish was treated with a $5 \mathrm{mg} / \mathrm{mL}$ BSA solution in PBS buffer for 60 minutes. In such a case, the surface was negatively charged in the presence of the PBS buffer $(\mathrm{pH}=7.4)$. As culture plates are frequently treated to improve cell adhesion and spreading (Zeiger, Hinton, \& Van Vliet, 2013), control BSA coated surface represents a substrate where chondrocyte adhesion is partially inhibited.

\subsection{AFM Measurements}

\subsubsection{SCFS approach}

In AFM, a minute tip is used as a sensor, and a cantilever serves as a transducer to measure different properties, surface and force interactions between the tip and the sample by means of cantilever deflection signals. This optical signal can be converted to an electric signal by using a photodiode detector with quadrant phases and recorded on a computer. 
When the AFM cantilever is bent by an applied force during the scanning topography or force measurement, the angle of the deflected laser beam changes and is reflected onto the photodiode detector. The position of the laser spot moves on the photodetector, inducing voltaic signal changes. These signal changes can be read to quantitatively estimate cantilever bending and force. This technique allowed the investigation of the adhesion response of chondrocytes attached to tipless cantilevers on different chitosan supports using normal force measurement in the process depicted in figure 1.

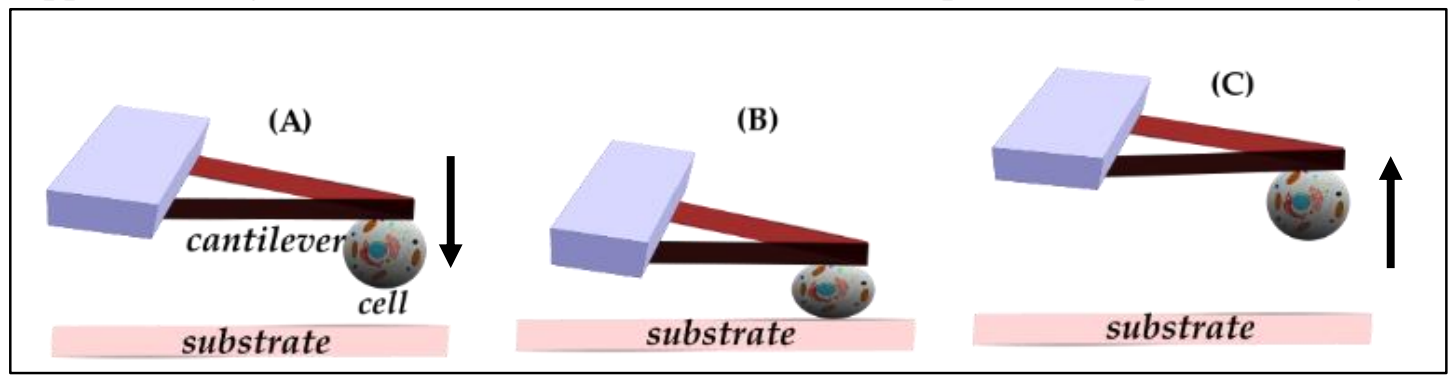

Figure 1. Global strategy for the cell adhesion measurements performed in this work. (A) Approach. Chondrocyte is attached to the cantilever and approached to the chitosan substrate at constant velocity. (B) Contact. Chondrocyte is in contact with the substrate during the contact time $\left(t_{c}\right)$ under force $\left(F_{c}\right)$. (C) Retraction. The cantilever is retracted and the cell interaction response is obtained.

The experiments were performed on a Nanowizard II AFM from JPK Instruments (Berlin, Germany). Soft tipless V-shaped commercial cantilevers MLCT-O (Bruker, France) with a spring constant $(\mathrm{k})$ around $0.01 \mathrm{~N} / \mathrm{m}$ were used to measure force strength. The spring constant was calibrated following a classical method, first the sensitivity $(\sim 50 \mathrm{~nm} / \mathrm{V})$ was found by contact on a rigid surface, then the method of thermal fluctuations (Hutter \& Bechhoefer, 1993) was used to find $\mathrm{k} \sim 0.01 \mathrm{~N} / \mathrm{m}$.

2.7.2 Single cell binding

(a)

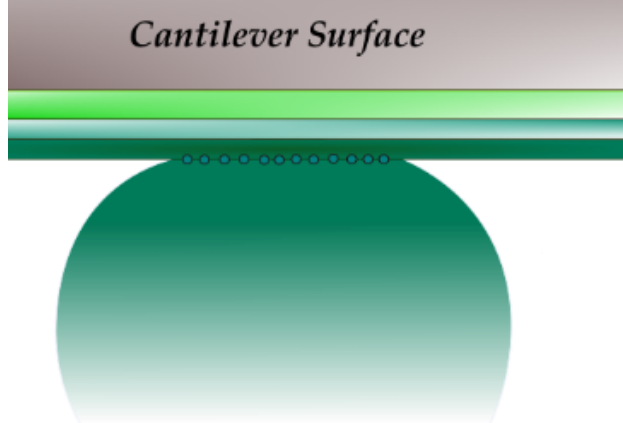

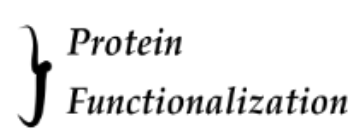

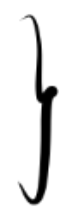

(b)

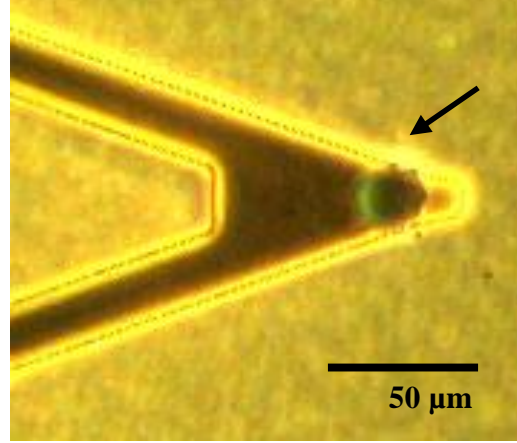

Figure 2. (a) Cantilever functionalization for cell attachment prior adhesion measurements. (b) Living chondrocyte adherent to the cantilever tip (as pointed by the black narrow) and placed on the top of chitosan film as substrate. The diameter of the chondrocyte determined by fluorescence is around $20 \mu \mathrm{m}$.

The global strategy consisted in the attachment of an individual chondrocyte cell, which was extracted from its original culture medium. The cantilever was pre-treated with different proteins allowing the binding of the cell to the cantilever tip, as it depicted in 
figure 2a. The cantilever functionalization consisted in the use of Biotin-BSA; an overnight treatment by incubation at $37^{\circ} \mathrm{C}$, followed by Streptavidin during 10 minutes under the same conditions, and the final step of the treatment involved the immersion of the tips into a Biotin-conA solution for 10 minutes (Laurent, Duperray, Sundar Rajan, \& Verdier, 2014; Sundar Rajan, Laurent, Verdier, \& Duperray, 2017). Intermediate cantilever rinsing with PBS between each step was carried out.

The chondrocyte was first captured, as shown in figure $2 \mathrm{~b}$, with the cantilever in $2 \mathrm{~mL}$ serum-free culture medium. Complete culture medium was added and the cell was then approached to the chitosan support which was fixed at the bottom part of the Petri dish. The force set point $\left(\mathrm{F}_{\mathrm{c}}\right)$ was selected to $0.5 \mathrm{nN}$ (applied force limit in the normal direction during the contact time) and the cantilever speed was $1 \mu \mathrm{m} / \mathrm{s}$.

As tipless cantilevers are used for this approach, the influence of the cell on the cantilever does not change the cantilever proprieties (in particular stiffness, $\mathrm{k}$ ), as shown previously (Laurent et al., 2014). The most important point is that the cell should be effectively in contact with the fiber mat or chitosan film which is the case according to the force curves obtained by microscopy.

\subsubsection{Analysis of AFM cell response}

The system response to the AFM experimental procedure consists in two curves corresponding to the approach and retraction processes. Vertical force $\mathrm{F}(\mathrm{nN})$ of the cantilever is represented versus piezo-height $(\mathrm{z})$. The piezoelectric device, placed at height $(\mathrm{z}) \sim 15 \mu \mathrm{m}$, moves from its position towards the bottom of the Petri dish until a vertical deflection according to the setpoint is observed. Once the contact time is achieved, the piezo retracts until the cell is completely detached from the substrate.

When the retraction region is analyzed, we are able to determine the number of significant adhesion events and the forces required to break each adhesion bond. This response could be directly related to the adherent protein distribution (a large family of integrins and CD44 receptors) among the cellular membrane that, especially in chondrocytes, mediate the capacity of the cell to have specific interactions with the ECM and regulate cartilage structure (Changhsun et al., 2008). Y a-t-il des protéines spécifiques? OUI, Mais les molécules d'adhésion sont sélectives. Les intégrines pour le collagène et le CD44 est un récepteur de HA.Et pour le chitosane ????

274 Each event, representing cell-substrate bond detachments, has a relative position (z) and intensity $(\Delta \mathrm{f})$. Figure 3 gives an example of a detachment step location (also called force jump), $\Delta \mathrm{f} \sim 70 \mathrm{pN}$, at $\mathrm{z}=1.55 \mu \mathrm{m}$ on the retraction curve. 


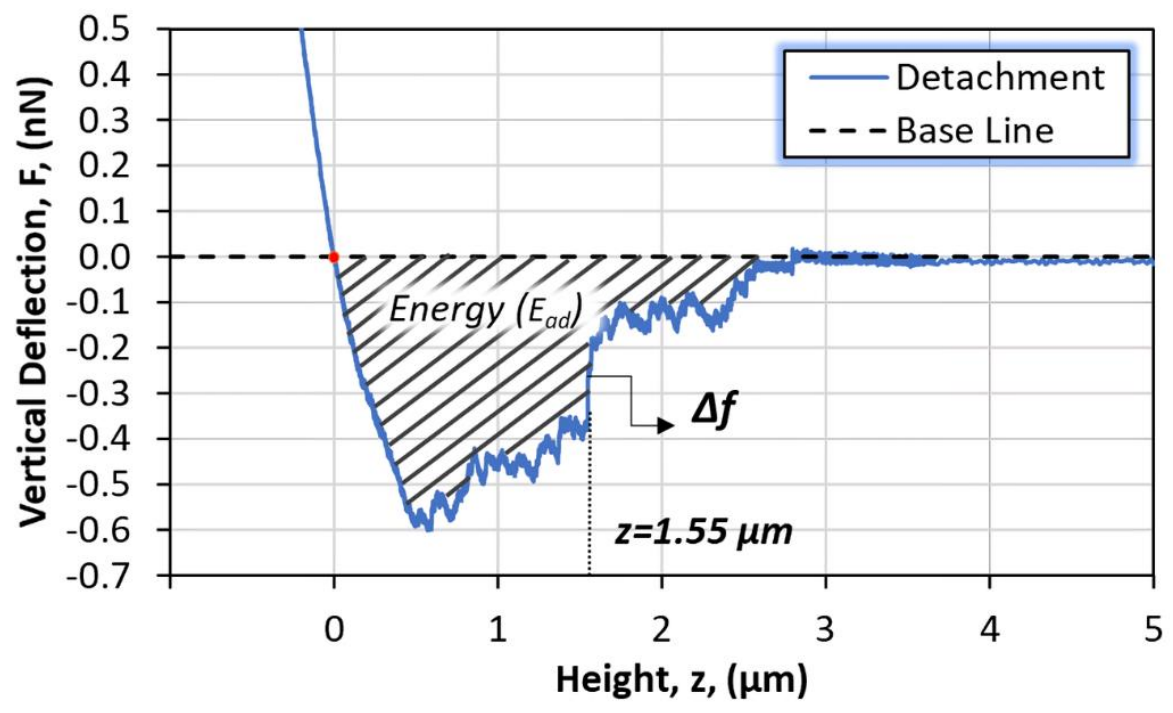

Figure 3. Retraction curve analysis. Force jump location (z) and intensity $(\Delta f)$ for adhesion test of chondrocytes on chitosan supports. Adhesion Energy (shaded area) represented as the integration of force (f) vs. cantilever displacement (z) for the detachment response of chondrocytes on the chitosan film. Contact time $=60 \mathrm{~s}$.

Another important aspect helping to characterize the interaction of chondrocytes to artificial scaffolds is the adhesion energy $\left(E_{a d}\right)$. The adhesion energy represents the detachment work done by the cantilever to completely detach the cell from the substrate. This parameter involves the whole cell contact area and is derived through integration of the area under the force $(\mathrm{nN})$ curve as a function of displacement $(\mathrm{z})$, presented in figure 3. In the same context as the other parameters studied, the base line is chosen as the final limiting value, where all bonds are considered detached (Laurent et al., 2014).

Tests were carried out at two different contact times (60 and 120 seconds) and two chitosan substrates (a casted film as model and an electrospun nanofiber mat with an average fiber diameter around 100-250 nm) (Garcia et al., 2018). A reference surface was prepared by coating the plastic of the Petri dish with BSA. It was determined that, under the same buffer conditions, zeta-potential indicates that cells are negatively charged.

\subsection{Statistical Analysis}

Data for adhesion assays were generated at three independent experiments, using around 15 contact points on each sample. All results are reported as mean with standard error of the mean (mean $\pm \mathrm{SE}$ ) as the error bar. The value $\mathrm{p}<0.01$ was considered statistically significant for comparison between sample groups, and it was obtained by one-way analysis of variance (ANOVA) using Excel.

\section{Results and discussion}

\subsection{Production of electrospun chitosan substrates}

Optimal conditions of electrospinning allowed the manufacturing of engineered chitosanbased fiber mats. For the electrospinning processed that was performed in the laboratory, aluminum foils cut in cross were chosen as support to remove the mat after processing. Fibers were observed to follow the stablished collector pattern as it is shown in figure 4a. 

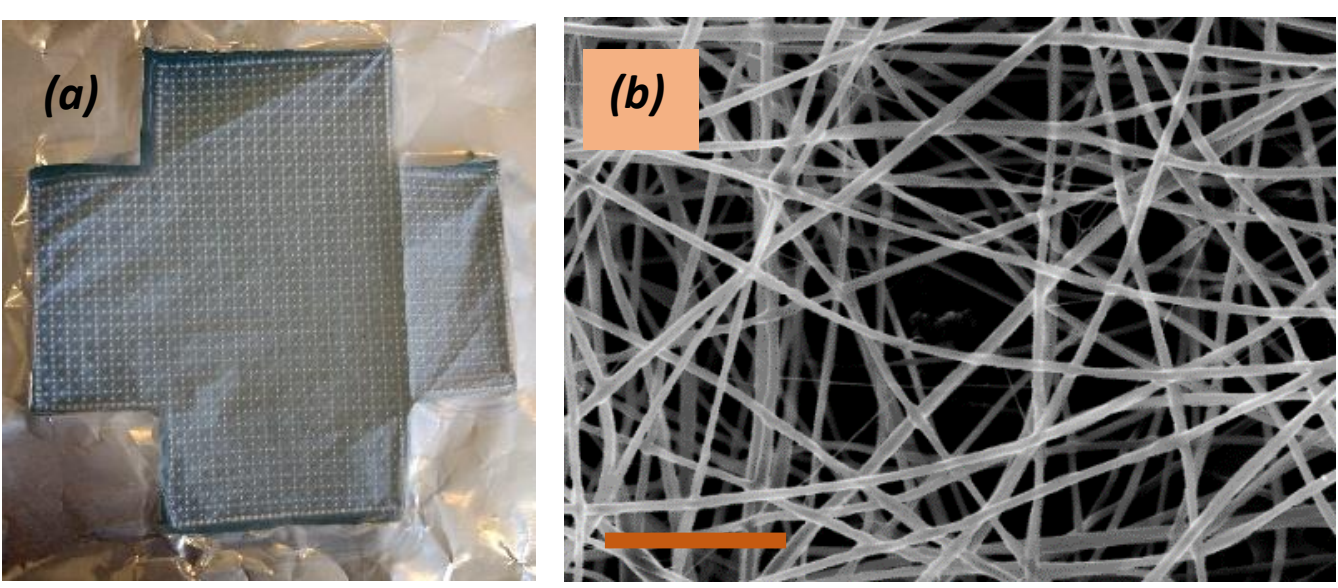

Figure 4. (a) Electrospun fiber mat recovered on squared-pattern collector. (b) As-spun porous fiber mat produced from 70/30 CS/PEO solution. Scale bar $=2 \mu \mathrm{m}$.

Morphology of electrospun chitosan fibers obtained under the same conditions has been analyzed. An average diameter of $118 \pm 36 \mathrm{~nm}$ was found after diameter distribution of the samples with a 70/30 CS/PEO proportion (Garcia et al., 2018). Smooth fibers and homogeneous mats were observed as it is shown in figure $4 \mathrm{~b}$.

\subsection{AFM response for force detachment}

The contact between the chondrocyte and the surface corresponds to indentation and adhesion interactions. After the prescribed contact time, the device retracts back, at a velocity of $1 \mu \mathrm{m} / \mathrm{s}$, to its initial position on the vertical axis. During this step, complete detachment of the cell occurs (in figure 5, read from left to right). In figure 5, the retraction curve obtained on chitosan films and fiber mats are compared with the BSA coated surface as reference.

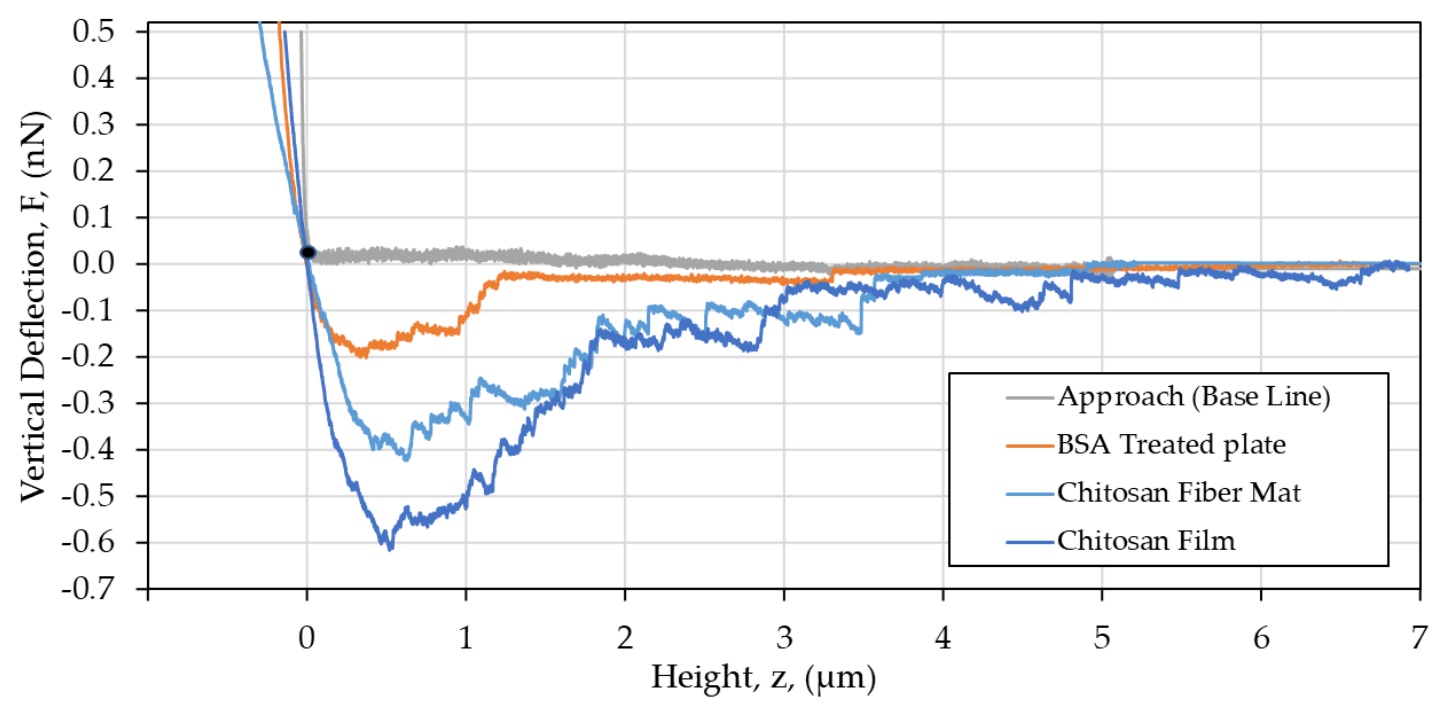

Figure 5. Comparative response of chondrocyte detachment on chitosan substrates (film and fiber mat) and coated Petri dish. The point $(0,0)$ on the curve F vs $\mathrm{z}$ represents the cell-substrate contact point. Retraction velocity of $1 \mu \mathrm{m} / \mathrm{s}$ and data shown for a contact time $\left(\mathrm{t}_{\mathrm{c}}\right)=60 \mathrm{~s}$.

326 Once the cell is in full contact with the substrate, the former is pushed towards the surface until the force set point is achieved. Herein, cell indentation occurs and, depending on the 
substrate properties, such as porosity, roughness, and swelling, a gap (in height) might be found between the approach and the retraction curves. In the same way for each material, this effect is observed in the different initial slopes on the detachment response $(F>0)$ (figure 5). Especially, on fiber mat with high porosity and higher water retention capacity (Garcia et al., 2018), the larger deviation (around $-0.25 \mu \mathrm{m}$ at $\mathrm{F}=0.5 \mathrm{nN}$ ) was detected.

In all retraction curves, having a similar trend, the different steps of cell detachment are identified and they provide a complementary understanding of cell adhesion measurements. In this regard, three regions can be differentiated, including all phenomena occurring, and are proposed in figure 6.

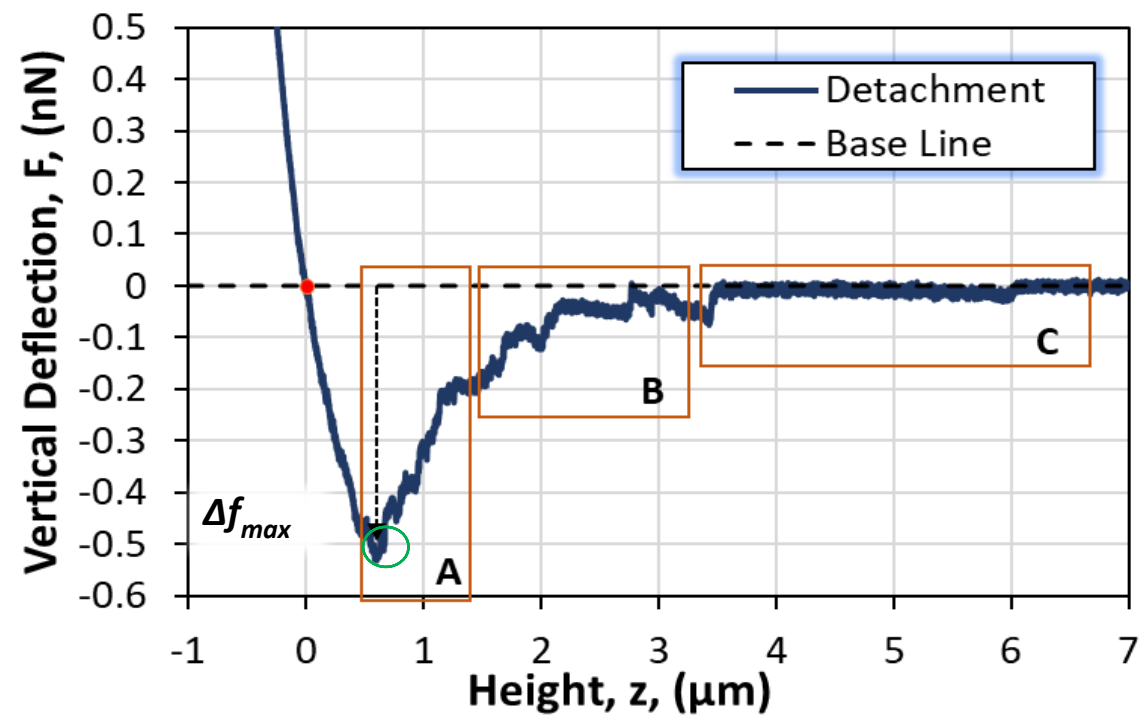

Figure 6. Cell detachment response separated in 3 regions or steps. Initial detachment in region $\mathrm{A}$, rupture of secondary cell-substrate bonds in zone $\mathrm{B}$ and breaking of remaining links and return to base line in region $\mathrm{C}$.

Included in the retraction curve, it is relevant to analyze the location and intensity of the maximum force value $\left(\Delta f_{\max }\right)$ observed on the detachment cell response (figure 6 ). This value is considered as the necessary detachment force to stretch the cell and the substrate (ECM in the native tissue) until cell-substrate bonds start to break.

In zone $A$, the highest vertical deflection value $\left(\Delta f_{\max }\right)$ is observed (green circle in figure 6 ) and marks the beginning of the detachment process; this peak can be associated to the cell-substrate assembly links being stretched at the same time and the point where they start to break. In this region, more than $60 \%$ of the detected force jumps $(\Delta f)$ occur in the next micrometer after the first breakup, this being considered as the more representative part of the detachment response.

As compared to zone A, a similar phenomenon appears in region B but the normal force decreases due to the reduction of bond number between the chondrocyte and support. Finally, in region $\mathrm{C}$, the final links are stretched and break as long as the cell is completely separated from the substrate surface, those links being more isolated could be associated with the individual response of cell membrane tethers (Sundar Rajan et al., 2017; Titushkin \& Cho, 2006). Tether length was found between $0.5 \mu \mathrm{m}$ and $1.5 \mu \mathrm{m}$ for both 
chitosan films and fiber mats. It is important to mention that a minority of the detected events in the retraction response take place in region $\mathrm{C}$.

Considering all the tests performed, the maximum vertical deflection values $\left(\Delta \mathrm{f}_{\max }\right)$ develop in the first micrometer of the retraction step (figure 6). The distribution of these forces comparing the chitosan film and the electrospun mat for a given contact time of 60 seconds is shown in figure 7 . From these results, a higher maximal normal deflection $\left(\Delta f_{\max }\right)$ is observed when the chondrocytes interact with a more compact surface (the chitosan film) in contrast with the porous fiber mat for which the $\Delta \mathrm{f}_{\max }$ values are smaller and more homogenous. In the same context, significant $\Delta \mathrm{f}_{\max }$ differences, when comparing CS substrates from the BSA coated surface, were found for $t_{c}=60 \mathrm{~s}$. This contrast between the studied substrates shows clearly that chondrocytes are around 2 times more strongly adhered to CS than when they are in contact with the coated BSA culture dish.

Average $\Delta \mathrm{f}_{\max }$ values between 2 and $7 \mathrm{nN}$ were previously observed in adhesion tests for chondrocytes using a different AFM approach (Changhsun et al., 2008). This difference can be attributed mainly to the experimental AFM arrangement applied and the origin of the chondrocyte sample.

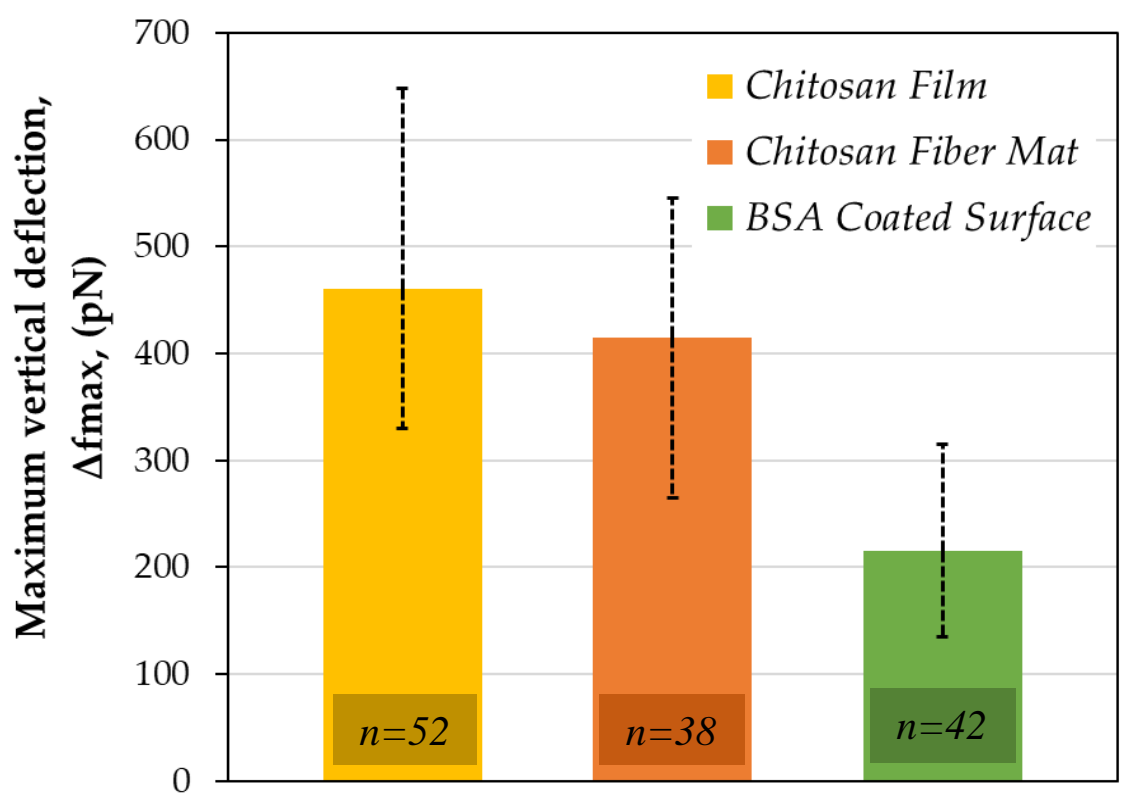

Figure 7. Distribution of maximum vertical force $\left(\Delta \mathrm{f}_{\max }\right)$ for the two substrates studied: chitosan film and chitosan nanofibers compared to the reference BSA coated surface (significant difference found, $\mathrm{p}<0.01$ ), for a contact time of $60 \mathrm{~s}$. Standard deviation in dotted line, number of assays $=\mathrm{n}$.

Because the force jump intensities are coupled with a relative position on the retraction curve, the distribution of detachment steps as a function of the location on the vertical axis (separation distance) can help understand the complete adhesion phenomenon. Towards that end, registered force jumps on chitosan substrates are presented in figure 8 , showing an important concentration of events during the initial part of the cell adhesion response attributed to the breakup of a large quantity of formed links and slight cell membrane deformation (zones A and B). Few force jumps are observed before complete 
detachment of the cell, those bonds could be related to a more complex interaction between the cellular membrane and the substrates. As the cell membrane is connected to the cytoskeleton, when the former detaches, cytoskeleton filaments (membrane tethers) are also elongated few micrometers until they are released (Sundar Rajan et al., 2017; Titushkin \& Cho, 2006).OK
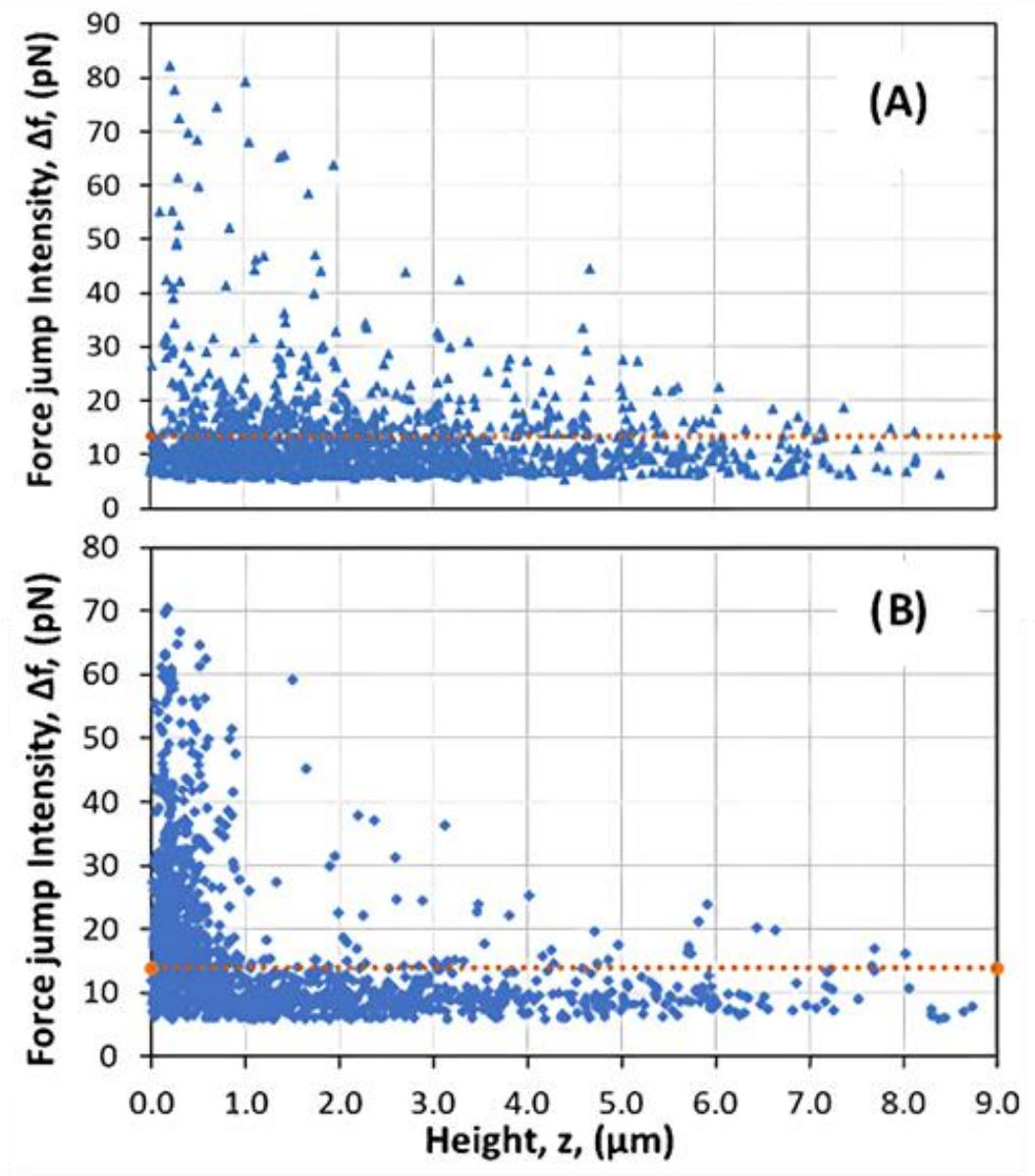

411 Figure 8. Distribution of force jumps $(\Delta \mathrm{f})$ for chondrocyte detachment from chitosan 412 substrates vs. height $(\mu \mathrm{m})$ starting from $\Delta \mathrm{f}$ max. Contact time of 60 seconds on the (A) 413 chitosan film and (B) chitosan fiber mat. Dotted lines represent the average detachment force values for the film and the fibers, $13.37 \mathrm{pN}$ and $13.85 \mathrm{pN}$, respectively.

415 As shown in figure 5 and described in figure 8, force jumps are randomly located along the retraction curve. There are no specifications about the order of every detachment step but they all can be associated with the rupture, at different steps, of chondrocyte-tosubstrate links formed during the pause interval when the cell membrane enters in contact with the substrate.

Expliquer pourquoi c'est moins disperse pour les fibres ????

421 Considering the final straight line as the base line for data analysis (figure 5), the retraction response of cells (figure 8 ) enables to build a frequency distribution curve of force jumps $(\Delta \mathrm{f})$, as shown in figure 9 . This analysis groups in intervals all significant $\Delta \mathrm{f}$ values, and allows to find the force jump average that could be related to an individual cell-substrate bond breaking. 


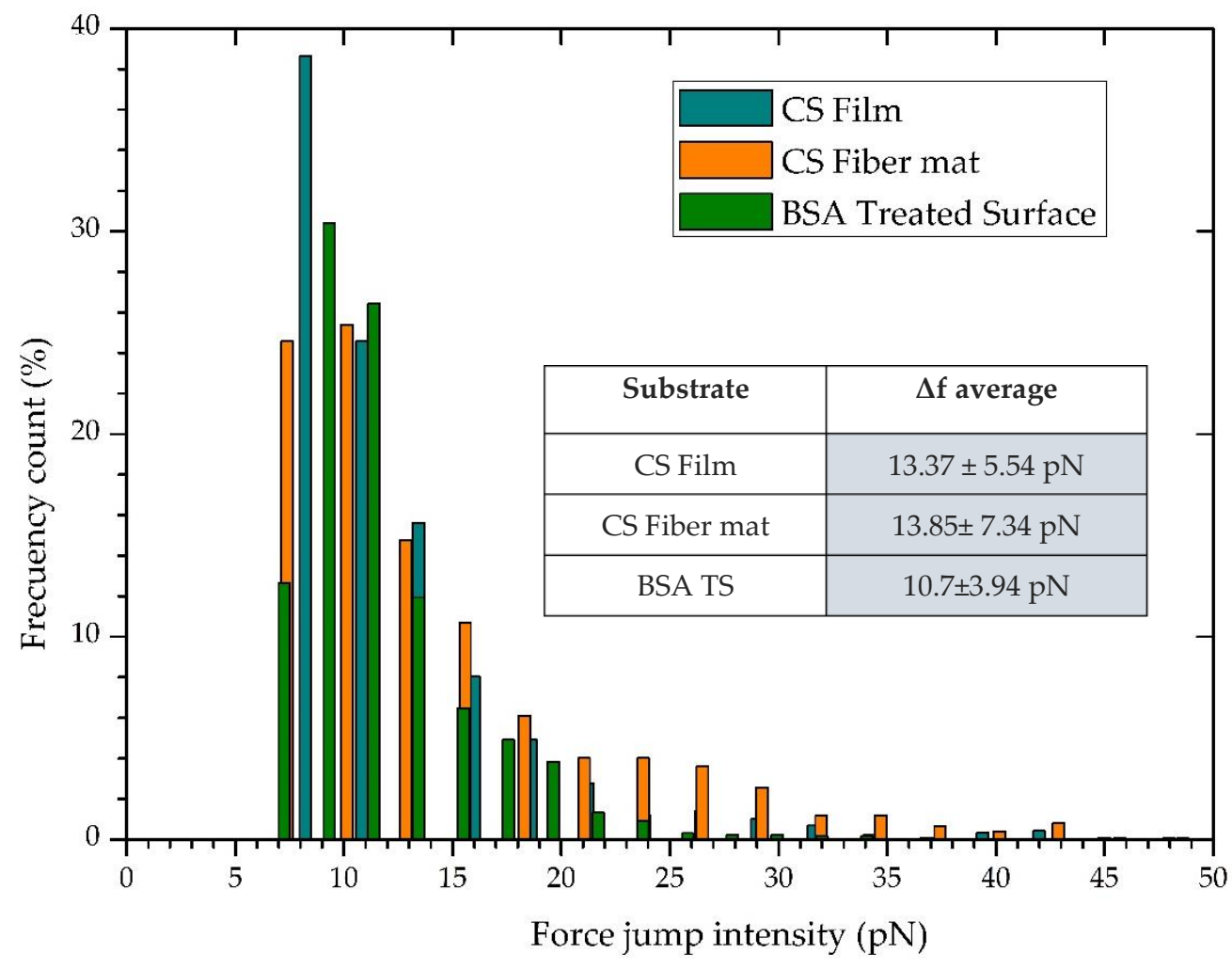

Force jump intensity $(\mathrm{pN})$

427 Figure 9. Force jump distribution for cell detachment on a chitosan fiber mat for a contact time of 60 seconds. Force jumps for CS films and the BSA coated surface are presented in the table for a similar $t_{c}$.

430 Cell detachment force jumps $(\Delta \mathrm{f})$ for chitosan substrates were contrasted taking into account that frequency distributions for different contact times have a similar distribution trend. This comparison is shown in figure 8, in terms of morphology (film and fiber) as well as in terms of affinity (BSA coated surface) for a contact time of $60 \mathrm{~s}$. In the case of a contact time of 120 seconds, there were found $14.73 \pm 7.58 \mathrm{pN}$ and $13.97 \pm 6.14 \mathrm{pN}$ for CS film and fiber mat, respectively. From the obtained main values in figure 8 , it is observed that the contact time does not reflect a significant difference $(p>0.01)$ on the average detachment steps $(\Delta \mathrm{f})$ between the studied chitosan-based substrates, independently of their morphology. This similarity could be explained for chitosan fibers and films, since the cell type is the same in all cases and ligand-receptor interactions have the same nature (CS-Chondrocyte).

441 It can be also remarked that the average $\Delta \mathrm{f}$ is higher for the chitosan films and fibers compared to the BSA coated surface (as significant difference was found for a $\mathrm{p}<0.01$ ANOVA analysis), an effect that can be attributed to the low cell-substrate selective interaction unfavored by BSA.

For the adhesion response, using the same technique for cell-cell adhesion strength, between an endothelial cell monolayer and tumor cells, detachment steps have been measured between 20 and 70 pN (Laurent et al., 2014; Sundar Rajan et al., 2017). These reference values are in the same range with the obtained response in the present experiments (detachment jumps between 10 and $80 \mathrm{pN}$ in figure 7). Values acquired from different variants of AFM methods consisting of lateral displacement or detachment from 
a suction micropipette on different types of cells are usually larger than those obtained in this work (Sagvolden et al., 1999; Tsang et al., 2006).

\subsection{Adhesion Energy of chondrocytes on chitosan substrates}

The adhesion energy was investigated for both cell-substrate responses, the chitosan film and the fiber mat, for a contact time of 60 seconds and compared to the BSA coated surface. As shown in figure 10, this parameter was affected, as expected, by the substrate morphology. The average adhesion energy value when the chitosan film was used as chondrocyte support was found $27 \%$ higher the one observed for the nanofiber mat, $7.68 \times 10^{-16} \mathrm{~J}$ and $6.05 \times 10^{-16} \mathrm{~J}$, respectively. Moreover, this difference was shown to exhibit the same trend for maximum detachment force values $\left(\Delta \mathrm{f}_{\max }\right)$ which are slightly higher for the chondrocyte-film interaction (see figure 9). This could be attributed to the quantity of cell-substrate bonds that were formed during the contact time and are expressed on the detachment response. On the other hand, we must consider the available contact surface when the chondrocyte touches the substrate. Due to fiber mat porosity, stiffness (Zhang, Yu, \& Zhao, 2016) and morphology, a slightly smaller and softer direct area is offered for the cell to attach during the short contact with the substrate surface .

Furthermore, non-vertical bonds are more likely to appear on fibers than films. This phenomenon could result in a lower energy adhesion, $\Delta \mathrm{f}_{\max }$ values and, at the same time, affect the placement of force jumps $(\Delta \mathrm{f})$ (figure 8$)$ on the retraction curve.

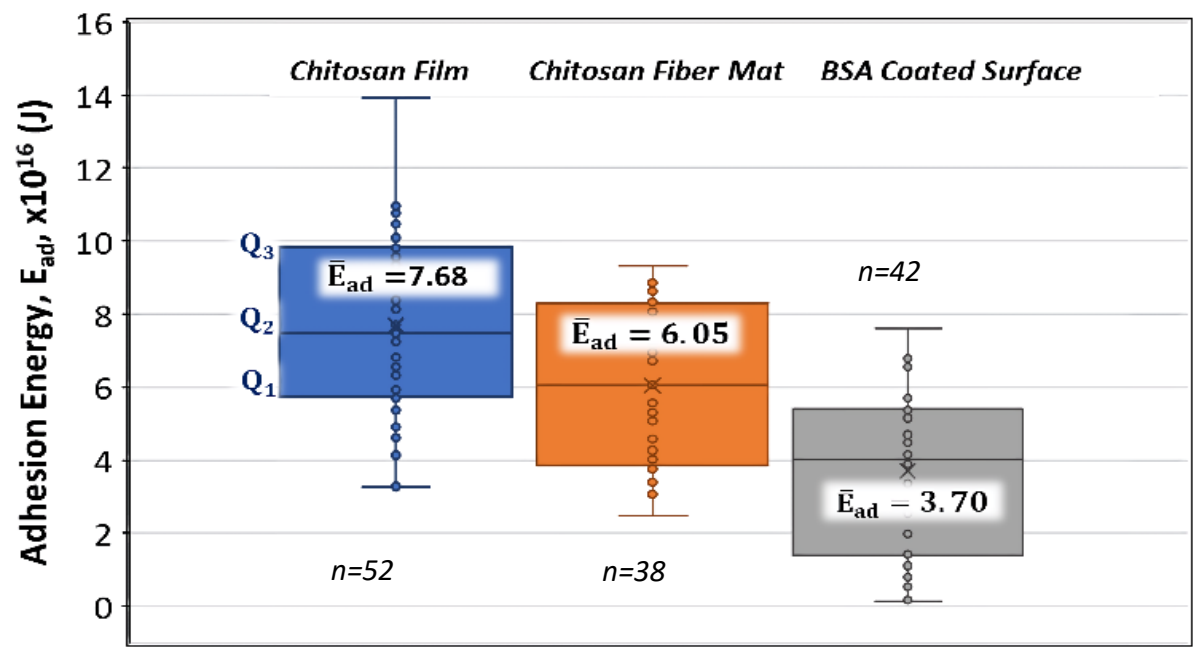

Figure 10. Adhesion Energy $\left(E_{a d}\right)$ distribution for AFM adhesion test on chitosan-based substrates (chitosan film and chitosan fiber mat) compared to the BSA treated surface (significant difference found, $\mathrm{p}<0.01$ ). $25 \%$ of the data being lower and higher than first $\left(Q_{1}\right)$ and third $\left(Q_{3}\right)$ quartiles, respectively, are out of the box plot. The average value is represented with the cross in the colored area. Contact time equal to $60 \mathrm{~s}$, number of assays $=n$.

Finally, it is important to compare the adhesion parameters on chitosan to that obtained for the surface coated with BSA (figure 4). The maximum $\Delta \mathrm{f}$ for chondrocytes in contact with a BSA-treated surface had a main value of $223 \mathrm{pN} \pm 99 \mathrm{pN}$. This response is significantly lower compared to the chitosan film and fibers $(\mathrm{p}<0.01)$. In addition, the adhesion energy value remains clearly lower than the response observed for the chitosanbased nanofiber mat and much lower than the detachment response in the case of the 
chitosan film $(\mathrm{p}<0.01)$. The average adhesion energy determined is $3.70 \times 10^{-16} \mathrm{~J} \pm$ $2.18 \times 10^{-16} \mathrm{~J}$ for the BSA-treated surface.

The effectiveness of the BSA coating was confirmed by the application of the same AFM procedure on a culture dish utilized as bought. After force measurement, adhesion energy was found to be $13.23 \times 10^{-16} \mathrm{~J} \pm 3.94 \times 10^{-16} \mathrm{~J}$. Such a high value is expected since commercial polystyrene plates and flasks are treated to render the surfaces hydrophilic, enhancing cell-substrate adhesion (Zeiger et al., 2013).

From the other hand, in the buffer used, BSA coated surface is negatively charged which promotes slight electrostatic repulsion as chondrocyte membrane has also a negative character. Cell adhesion on chitosan substrates is favored due to $\mathrm{H}$-bond stabilization, hydrophilicity and polarity which serves to bind proteins on its surface. Protein adsorption and subsequent cell adhesion on biomaterial surface is the essential prerequisite for biomaterial induced tissue healing (Sukul et al., 2021).

\section{Conclusions}

The cell adhesion study revealed that the adhesive response depends largely on the environmental properties of the chitosan-based materials (different morphology and surface). In this context, it was observed a slightly higher adhesion for the chitosan film compared to the chitosan fiber mat. This response can be explained considering the quantity of cell-substrate bonds that could be formed in the larger contact surface offered, in this case, by the chitosan film in contact with the cell membrane. Such bonds lead to a higher detachment force and adhesion energy values even for short contact times. Whatever the chitosan substrate used, the adhesion is favored compared to a negative BSA coated surface; a difference that involves $\mathrm{H}$-bond and electrostatic loose contribution between chondrocyte and chitosan. Additionally, the porous nanofiber mat should allow cell migration, nutriment transport and permeability.

To our knowledge, no experimental data on SCFS have been published for chondrocytes on different CS substrates. Data from the adhesive responses presented here, allow to validate our proposed hypothesis showing that chitosan-based nanofiber mats are the most convenient supports compared with homogeneous films for chondrocyte proliferation applied in tissue engineering.

\section{Conflicts of interest}

There are no conflicts to declare.

\section{Author Contributions}

C.V., C.E.G.G. and M.R. conceived and designed the experiments; C.E.G.G. and C.V. performed the experiments; C.E.G.G., C.V., F.A.S.M., M.R., B.L. and F.B. analyzed the data and wrote the paper. All authors have read and agreed to the published version of the manuscript.

\section{Funding Sources}

This work has been financially supported by Mexican CONACYT grant 611845/788990 attributed to Christian E. García García to prepare a $\mathrm{PhD}$ thesis in cooperation between LRP (University Grenoble-Alpes, France) and CUCEI (University of Guadalajara, 
Mexico). We thank the Nanoscience foundation for financial support regarding the AFM platform at Liphy.

\section{Acknowledgements}

The authors acknowledge H.L. Lauzon from Primex Ehf (Iceland) for the gift of the chitosan sample and D. Roux from LRP (UGA) for his scientific interest and collaboration. LIPhy and LRP are members of the LabeX Tec 21 (Investissements d'Avenir: grant agreement No. ANR-11-LABX-0030).

\section{References}

Amaral, I. F., Cordeiro, A. L., Sampaio, P., \& Barbosa, M. A. (2007). Attachment, spreading and short-term proliferation of human osteoblastic cells cultured on chitosan films with different degrees of acetylation. Journal of Biomaterials Science, Polymer Edition, 18(4), 469-485. https://doi.org/10.1163/156856207780425068

Changhsun, H., Y.-H., L., Shuming, L., Jyy-Jih, T.-W., Chung Hsiun, H. W., \& ChingChuan, J. (2008). Surface ultrastructure and mechanical property of human chondrocyte revealed by atomic force microscopy. Osteoarthritis and Cartilage, 16(4), 480-488. https://doi.org/10.1016/j.joca.2007.08.004

Cohen, M., Klein, E., Geiger, B., \& Addadi, L. (2003). Organization and adhesive properties of the hyaluronan pericellular coat of chondrocytes and epithelial cells. Biophysical Journal, 85(3), 1996-2005. https://doi.org/10.1016/S00063495(03)74627-X

Garcia, C. E., Soltero Martínez, F. A., Bossard, F., \& Rinaudo, M. (2018). Biomaterials based on electrospun chitosan. Relation between processing conditions and mechanical properties. $\quad$ Polymers, 10(3), 1-19. https://doi.org/10.3390/polym10030257

Goldring, M. B., Birkhead, J. R., Suen, L., Yamin, R., Mizuno, S., Glowacki, J., ... Apperleyll, J. F. (1994). Interleukin-1,8-modulated Gene Expression in Immortalized Human Chondrocytes. J Clin Invest, 94(6), 2307-2316.

Huang, B. J., Hu, J. C., \& Athanasiou, K. A. (2016). Cell-based tissue engineering strategies used in the clinical repair of articular cartilage. Biomaterials, 98, 1-22. https://doi.org/10.1016/j.biomaterials.2016.04.018

Hutter, J. L., \& Bechhoefer, J. (1993). Calibration of atomic-force microscope tips. Review of Scientific Instruments, 64(7), 1868-1873. https://doi.org/10.1063/1.1143970

Iscru, D. F., Anghelina, M., Agarwal, S., \& Agarwal, G. (2008). Changes in surface topologies of chondrocytes subjected to mechanical forces: An AFM analysis. Journal of Structural Biology, 162(3), 397-403. https://doi.org/10.1016/j.jsb.2008.02.005

Laurent, V. M., Duperray, A., Sundar Rajan, V., \& Verdier, C. (2014). Atomic Force Microscopy Reveals a Role for Endothelial Cell ICAM-1 Expression in Bladder Cancer Cell Adherence. PLOS ONE, 9(5), 1-11. https://doi.org/10.1371/journal.pone.0098034

Nguyen, T. D., \& Gu, Y. (2016). Investigation of Cell-Substrate Adhesion Properties of 
Living Chondrocyte by Measuring Adhesive Shear Force and Detachment Using AFM and Inverse FEA. Scientific Reports, 6(38059), 1-13. https://doi.org/10.1038/srep38059

Puech, P. H., Taubenberger, A., Ulrich, F., Krieg, M., Muller, D. J., \& Heisenberg, C. P. (2005). Measuring cell adhesion forces of primary gastrulating cells from zebrafish using atomic force microscopy. Journal of Cell Science, 118(18), 4199-4206. https://doi.org/10.1242/jcs.02547

Rai, V., Dilisio, M. F., Dietz, N. E., \& Agrawal, D. K. (2017). Recent strategies in cartilage repair: A systemic review of the scaffold development and tissue engineering. Journal of Biomedical Materials Research - Part A, 105(8), 23432354. https://doi.org/10.1002/jbm.a.36087

Ribba, L., Parisi, M., D’Accorso, N. B., \& Goyanes, S. (2014). Electrospun nanofibrous mats: From vascular repair to osteointegration. Journal of Biomedical Nanotechnology, 10(12), 3508-3535. https://doi.org/10.1166/jbn.2014.2046

Rinaudo, M. (2006). Chitin and chitosan: Properties and applications. Progress in Polymer Science (Oxford), 31(7), 603-632. https://doi.org/10.1016/j.progpolymsci.2006.06.001

Sagvolden, G., Giaever, I., Pettersen, E. O., \& Feder, J. (1999). Cell adhesion force microscopy. Proceedings of the National Academy of Sciences of the United States of America, 96(2), 471-476. https://doi.org/10.1073/pnas.96.2.471

Sapkota, S., \& Chou, S. (2020). Electrospun Chitosan-based Fibers for Wound Healing Applications, 4(2), 51-57. https://doi.org/10.11648/j.jb.20200402.13

Soliman, S., Sant, S., Nichol, J. W., Khabiry, M., Traversa, E., \& Khademhosseini, A. (2011). Controlling the porosity of fibrous scaffolds by modulating the fiber diameter and packing density. Journal of Biomedical Materials Research - Part A, 96 A(3), 566-574. https://doi.org/10.1002/jbm.a.33010

Sukul, M., Sahariah, P., Lauzon, H. L., Borges, J., Másson, M., Mano, J. F., ... Reseland, J. E. (2021). In vitro biological response of human osteoblasts in 3D chitosan sponges with controlled degree of deacetylation and molecular weight. Carbohydrate Polymers, 254(November). https://doi.org/10.1016/j.carbpol.2020.117434

Sundar Rajan, V., Laurent, V. M., Verdier, C., \& Duperray, A. (2017). Unraveling the Receptor-Ligand Interactions between Bladder Cancer Cells and the Endothelium Using AFM. Biophysical Journal, 112(6), 1246-1257. https://doi.org/10.1016/j.bpj.2017.01.033

Titushkin, I., \& Cho, M. (2006). Distinct Membrane Mechanical Properties of Human Mesenchymal Stem Cells Determined Using Laser Optical Tweezers. Biophysical Journal, 90(7), 2582-2591. https://doi.org/10.1529/biophysj.105.073775

Tsang, P. H., Li, G., Brun, Y. V, Freund, L. Ben, \& Tang, J. X. (2006). Adhesion of single bacterial cells in the micronewton range. Proceedings of the National Academy of Sciences of the United States of America, 103(15), 5764-5768. https://doi.org/10.1073/pnas.0601705103

Ungai-Salánki, R., Peter, B., Gerecsei, T., Orgovan, N., Horvath, R., \& Szabó, B. (2019). 
A practical review on the measurement tools for cellular adhesion force. Advances in Colloid and Interface Science. https://doi.org/10.1016/j.cis.2019.05.005

Varady, N. H., \& Grodzinsky, A. J. (2016). Osteoarthritis year in review 2015: Mechanics. Osteoarthritis and Cartilage, 24(1), 27-35. https://doi.org/10.1016/j.joca.2015.08.018

Whitehead, K. A., Rogers, D., Colligon, J., Wright, C., \& Verran, J. (2006). Use of the atomic force microscope to determine the effect of substratum surface topography on the ease of bacterial removal. Colloids and Surfaces B: Biointerfaces, 51(1), 4453. https://doi.org/10.1016/j.colsurfb.2006.05.003

Yamane, S., Iwasaki, N., Majima, T., Funakoshi, T., Masuko, T., Harada, K., ... Nishimura, S. I. (2005). Feasibility of chitosan-based hyaluronic acid hybrid biomaterial for a novel scaffold in cartilage tissue engineering. Biomaterials, 26(6), 611-619. https://doi.org/10.1016/j.biomaterials.2004.03.013

Younes, I., \& Rinaudo, M. (2015). Chitin and chitosan preparation from marine sources. Structure, properties and applications. Marine Drugs, 13(3), 1133-1174. https://doi.org/10.3390/md13031133

Zeiger, A. S., Hinton, B., \& Van Vliet, K. J. (2013). Why the dish makes a difference: Quantitative comparison of polystyrene culture surfaces. Acta Biomaterialia, 9(7), 7354-7361. https://doi.org/10.1016/j.actbio.2013.02.035

Zhang, Q., Yu, Y., \& Zhao, H. (2016). The effect of matrix stiffness on biomechanical properties of chondrocytes. Acta Biochimica et Biophysica Sinica, 48(10), 958-965. https://doi.org/10.1093/abbs/gmw087 